\title{
ORIGINAL ARTICLE Incidence of respiratory viral infection in infants with respiratory symptoms evaluated for late-onset sepsis
}

\author{
JB Cerone ${ }^{1}$, RP Santos ${ }^{2}$, D Tristram ${ }^{2}$, DM Lamson ${ }^{3}$, KA Stellrecht ${ }^{4}, \mathrm{~K} \mathrm{St} \mathrm{George}^{3}$, MJ Horgan ${ }^{1}$ and A Rios $^{1}$
}

OBJECTIVE: To determine the frequency, etiology and impact of respiratory viral infection (RVI) on infants evaluated for late-onset sepsis (LOS), defined as sepsis occurring $>72 \mathrm{~h}$ of life, in the neonatal intensive care unit.

STUDY DESIGN: Prospective observational study conducted from 6 March 2014 to 3 May 2016 on infants evaluated for LOS. PCR viral panel performed on nasopharyngeal specimens among infants with clinical suspicion for RVI. Sequence analysis was performed to determine viral subtypes. Fisher's exact or $X^{2}$ tests were done to determine the impact of RVI.

RESULTS: During the 26-month study, there were 357 blood cultures obtained for LOS evaluations, 29 (8\%) had a respiratory virus detected. Only 88 (25\%) of infants evaluated for LOS also had clinical suspicion for a respiratory viral infection. RSV (14 of 29; $48 \%$ ) was the predominant virus detected. Almost all infants (13 of 14; 93\%) with RSV required increased respiratory support. Antimicrobial therapy was withheld or discontinued on most infants with a virus detected $(18$ of $29 ; 62 \%)$ and in the majority where there was no confirmed bacterial

co-infection (18 of 20 ; $90 \%$ ).

CONCLUSION: The incidence of RVI in infants being evaluated for LOS is about $8 \%$. RVI should be considered in LOS evaluation to prevent unnecessary antibiotic therapy.

Journal of Perinatology (2017) 37, 922-926; doi:10.1038/jp.2017.69; published online 18 May 2017

\section{INTRODUCTION}

Respiratory viral infection (RVI) may contribute to significant morbidity and mortality among infants presenting with sepsis. Significant illness in both preterm ${ }^{1}$ and full-term ${ }^{2}$ infants have been described with RVI. Several respiratory viral pathogens, including coronaviruses, enterovirus, human metapneumovirus, influenza, parainfluenza, respiratory syncytial virus (RSV) and rhinovirus have been reported as possible causes of late-onset sepsis (LOS). ${ }^{3-5}$ These viruses are associated with increased length of hospital stay, severe disease, unnecessary antimicrobial exposure and nosocomial outbreaks in the neonatal intensive care unit (NICU). ${ }^{5-7} \mathrm{RVI}$ is often underdiagnosed, unrecognized or infrequently examined as a cause of sepsis in infants because of several challenges: the wide range of clinical presentations and their similarity to bacterial infections ${ }^{8}$ and the unreliable methodology previously used for RVI detection which lacked sensitivity and specificity. ${ }^{3}$

The introduction of new molecular-based assays has paved the way for accurate detection of viral pathogens as a cause of sepsis in infants. The use of multiplex polymerase chain reaction (PCR) technology allows for prompt and more reliable detection of viral infection. ${ }^{3-5,9,10}$ Clinical suspicion for $\mathrm{RVI}$ promotes the detection of viral pathogens ${ }^{4}$ using PCR technology, enhancing early recognition and facilitating the initiation of appropriate antiviral treatment ${ }^{11}$ or prophylactic regimens. ${ }^{3}$ Furthermore, it facilitates the withholding or discontinuation of unnecessary antimicrobial therapy, contributing to
NICU-specific stewardship initiatives ${ }^{12}$ as well as outbreak prevention. $^{5}$

The objective of this study was to determine the frequency, etiology and impact of RVI on term and preterm infants evaluated for LOS in the NICU with clinical suspicion for viral infection.

\section{METHODS}

This was an IRB-approved single-center prospective observational study from 6 March 2014 to 3 May 2016 conducted in the NICU at the Bernard and Millie Duker Children's Hospital at Albany Medical Center. Our NICU is a 60-bed (private room) level IV regional perinatal center with $\sim 800$ annual admissions, $\sim 150$ with a birthweight below $1500 \mathrm{~g}$. The study population included any infant admitted to the NICU for LOS evaluation with an associated concern for RVI, regardless of gestational age or medical and surgical comorbidities. Admissions to the NICU include all inborn infants less than 35 weeks' gestation, infants transferred from another facility within our region, recently discharged preterm infants and infants greater than 35 weeks' with significant illness requiring intensive care up to 30 days of life. Each episode of LOS was counted individually. Infants evaluated for sepsis had a CBC and blood culture was obtained as well as additional tests such as a C-reactive protein (CRP), cerebral spinal fluid (CSF) studies, tracheal aspirate or urine cultures deemed necessary by the medical team based on the infant's clinical presentation. For infants with clinical suspicion for RVI, a nasopharyngeal specimen was obtained for PCR testing. The caregiver's clinical suspicion of viral infection had been described previously as the best predictor of RVI and respiratory signs indicative of infection had been defined extensively elsewhere. ${ }^{4}$ The PCR test was ordered at the discretion of the clinical team as well as empiric

${ }^{1}$ Department of Pediatrics/Division of Neonatology, Albany Medical Center, Albany, NY, USA; ${ }^{2}$ Department of Pediatrics/Division of Infectious Disease at Albany Medical Center, Albany, NY, USA; ${ }^{3}$ Laboratory of Viral Diseases at Wadsworth Center, New York State Department of Health, Albany, NY, USA and ${ }^{4}$ Department of Pathology and Laboratory Medicine at Albany Medical Center, Albany, NY, USA. Correspondence: Dr JB Cerone, Division of Neonatology, Albany Medical College, 43 New Scotland Ave, Mail Code 101, Albany, NY 12866, USA.

E-mail: ceronej@mail.amc.edu

Presented in part at the 2015 Pediatric Academic Societies Annual Meeting, San Diego, CA, 28 April 2015 (No. 429).

Received 21 December 2016; revised 19 March 2017; accepted 6 April 2017; published online 18 May 2017 


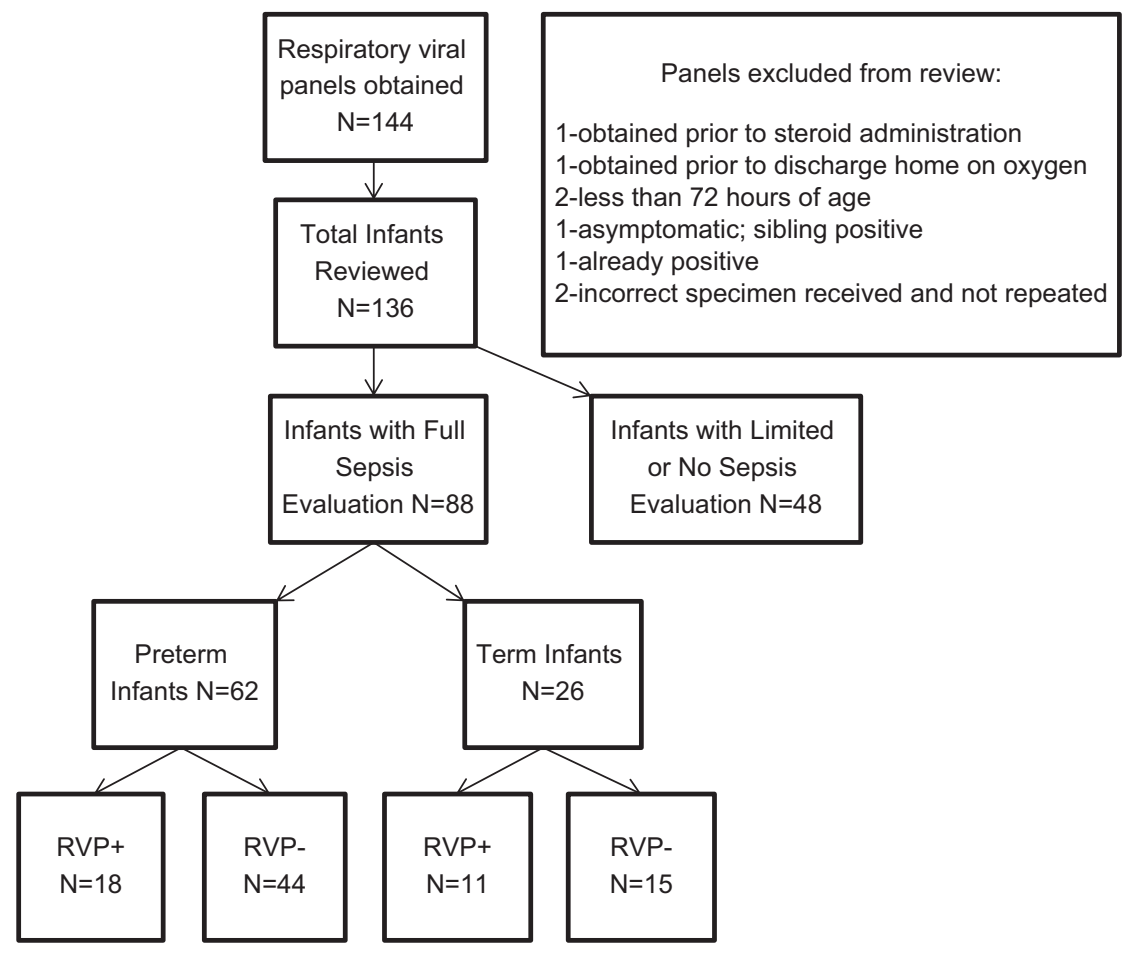

Figure 1. Flow diagram of study participants. RVP+, respiratory viral panel positive; RVP-, respiratory viral panel negative.

Table 1. Baseline case characteristics and demographics

\begin{tabular}{lccc}
\hline & $R V P+(\mathrm{N}=29)$ & $\left.R^{2}\right)$ \\
\hline Gestational age (weeks) & $343 / 7(23-41)$ & $314 / 7(23-41)$ & $\left.169^{\mathrm{a}}\right)$ \\
Birthweight (grams) & $2180(530-3860)^{*}$ & $1636(450-4110)$ & $0.03^{\mathrm{b}}$ \\
Sex (\% male) & $16(55 \%)$ & $32(60 \%)$ & $0.02^{\mathrm{b}}$ \\
Inborn/transfer (during birth hospitalization) & $6(33 \%)$ & $36(68 \%)$ & $0.72^{\mathrm{c}}$ \\
Diagnosis of chronic lung disease (CLD) & $3(10 \%)$ & $21(40 \%)$ & $<0.01^{\mathrm{c}}$ \\
Sepsis evaluation (day of life) & $38(8-101)$ & $52(4-222)$ & $<0.01^{\mathrm{c}}$ \\
\hline
\end{tabular}

Abbreviations: RVP+, respiratory viral panel positive; RVP-, respiratory viral panel negative. ${ }^{\text {a } F o r ~ c a l c u l a t i o n s ~ i n v o l v i n g ~ b i r t h ~ d e m o g r a p h i c s ~ i n f a n t ~ o n l y ~}$ counted once $(n=53)$. ' Unpaired $t$-test. ${ }^{c} x^{2}$ test. *Birthweight unavailable for two full-term infants.

antimicrobial coverage pending bacterial culture and/or PCR test results for at least $48 \mathrm{~h}$.

The clinical order for testing was placed using the electronic medical record system. Consent was waived as the respiratory viral panel test was obtained as part of the patients' routine medical care based on the clinical suspicion for RVI by the primary medical team. The information was prospectively collected.

A fine-tipped flocked swab was inserted into the nostril and back to nasopharynx, left in place for a few seconds and removed slowly with a slight rotation over the surface of the posterior nasopharynx. The same swab was then inserted into the other nostril repeating the process. The swab was then immediately placed in viral transport media and transported to the lab on wet ice. Upon receipt in the lab, the specimen remained on ice until testing, after which time the specimen was frozen at $-80^{\circ} \mathrm{C}$. The Film Array multiplex PCR Respiratory Panel (Bio Fire Diagnostics, Inc., Salt Lake City, UT, USA) was utilized, which detects 17 viral pathogens; adenovirus, coronaviruses (HCoV-HKUI, HCoV-NL63, $\mathrm{HCoV}-229 \mathrm{E}$ and $\mathrm{HCoV}-\mathrm{OC} 43$ ), human metapneumovirus (hMPV), rhinovirus/enterovirus (RhV/EV), influenza $A$, influenza $A / H 1$, Influenza $A / H 1$ 2009, influenza A/H3, influenza $B$, parainfluenza 1-4 (PIV1-PIV4) and respiratory syncytial virus (RSV). ${ }^{13-15}$ Results were available within $2 \mathrm{~h}$. The overall sensitivity of the respiratory panel for all viruses is $97 \%$, while specificity is $100 \%{ }^{16}$ Specimens from patients with positive results were sent to the Wadsworth Center, New York State Department of Health (NYS $\mathrm{DOH}$ ) for sequence analysis to determine specific viral types and subtypes.

Viral sequence analysis or molecular typing of viruses

Total nucleic acid was extracted using an easyMAG instrument (bioMerieux) from respiratory swabs that tested positive for RhV/EV or RSV on the BioFire Film Array (bioMerieux). The RhV or EV type was determined by amplification and sequence analysis of the VP1 gene. ${ }^{17}$ While that for RSV-positive samples to subgroup RSV-A or RSV-B targeted a region of the $G$ gene. $^{18}$ Sequences were analyzed using the NCBI BLAST tool to determine the viral types and/or subtypes.

Statistical analysis

Sample size calculation using simple interactive statistical analysis showed that a sample size of 33 in each group (preterm 33, full-term 33) was large enough to detect a $9 \%$ difference between the groups. ${ }^{19}$ Descriptive analyses were used for characterization of demographics. Statistical tests $\left(x^{2}\right.$ or Fisher's exact test) were used to analyze the categorical data, including the incidence of RVI in preterm versus full-term infants, severity of disease and discontinuation of antibiotic therapy in the presence of a positive viral PCR panel. All statistical analyses were performed using 
Table 2. Symptoms prompting late-onset sepsis evaluation

\begin{tabular}{lccr}
\hline & $R V P+(\mathrm{N}=29)$ & $R V P-(\mathrm{N}=59)$ & P-value \\
\hline Apnea & $13(45 \%)$ & $26(44 \%)$ & 0.95 \\
Fever & $7(24 \%)$ & $11(19 \%)$ & 0.55 \\
Cough & $9(31 \%)$ & $2(3 \%)$ & 0.02 \\
Congestion & $7(24 \%)$ & $3(5 \%)$ & $<0.01$ \\
Rhinorrhea & $2(7 \%)$ & $1(2 \%)$ & 0.46 \\
Secretions & $4(14 \%)$ & $13(22 \%)$ & 0.36 \\
Tachypnea & $2(7 \%)$ & $6(10 \%)$ & 0.62 \\
Increased work of breathing & $9(31 \%)$ & $7(12 \%)$ & 0.03 \\
Increased FiO2 & 0 & $12(20 \%)$ & $<0.01$ \\
Cyanosis & $6(21 \%)$ & $5(8 \%)$ & 0.10 \\
Poor feeding & $10(34 \%)$ & $4(7 \%)$ & $<0.01$ \\
Choking/gagging & 0 & $2(3 \%)$ & 0.32 \\
Abdominal distension & $1(3 \%)$ & $3(5 \%)$ & 0.73 \\
Neurologic & $1(3 \%)$ & $4(7 \%)$ & 0.53 \\
Lethargy & $5(17 \%)$ & $2(3 \%)$ & 0.02 \\
\hline
\end{tabular}

Abbreviations: FiO2, fraction of inspired oxygen; $\mathrm{RVP}+$, respiratory viral panel positive; RVP-, respiratory viral panel negative. All symptoms documented at time of evaluation were included. $x^{2}$ test used for comparison.

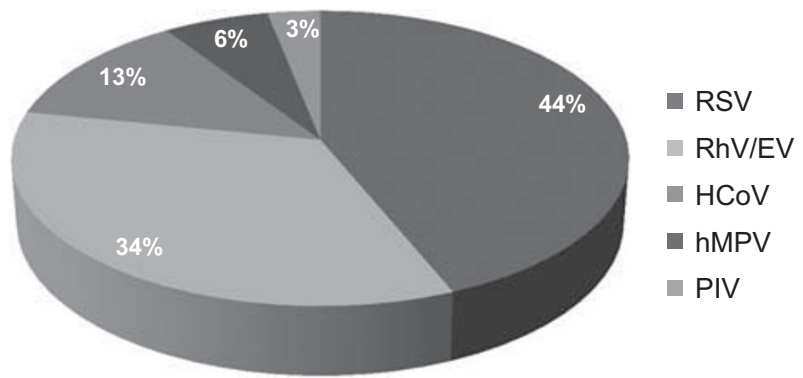

Figure 2. Respiratory viruses detected in infants undergoing LOS evaluation $\left(N=32^{*}\right)$, in order of decreasing frequency. RSV, Respiratory syncytical $(N=14) ; \mathrm{RhV}$, human rhinovirus; EV, enterovirus $(N=11)$; HCoV, human coronavirus $(N=4)$; hMPV, human metapneumovirus $(N=2)$; PIV, parainfluenzavirus $(N=1)$; 3 infants were co-infected with more than one virus.

Graphpad software (version 5.04, San Diego, CA, USA). A value of $a<0.05$ was considered statistically significant.

\section{RESULTS}

During the 26-month study period, 144 respiratory viral panels were obtained, of which 136 evaluations were reviewed and 88 $(61 \%)$ were included in the study (Figure 1). There were 62 preterm evaluations and 26 term evaluations included in the analysis. Eight infants were excluded from the review for reasons outlined in Figure 1 and 48 were not included with limited or no sepsis evaluation. During the study period 357 blood cultures were obtained for LOS evaluations; only $88(25 \%)$ of these evaluations included a respiratory viral panel. The demographics and baseline characteristics of infants included in the study are outlined in Table 1. There was no significant difference in gender or day of life in LOS evaluations for infants with or without a respiratory virus detected. There were, however, significant differences in gestational age, birthweight, evaluation during birth hospitalization and diagnosis of chronic lung disease. These differences may be attributed to the admission of older term infants and the readmission of recently discharged premature infants requiring intensive care support. Cough, nasal congestion, increased work of breathing, lethargy and poor feeding were the

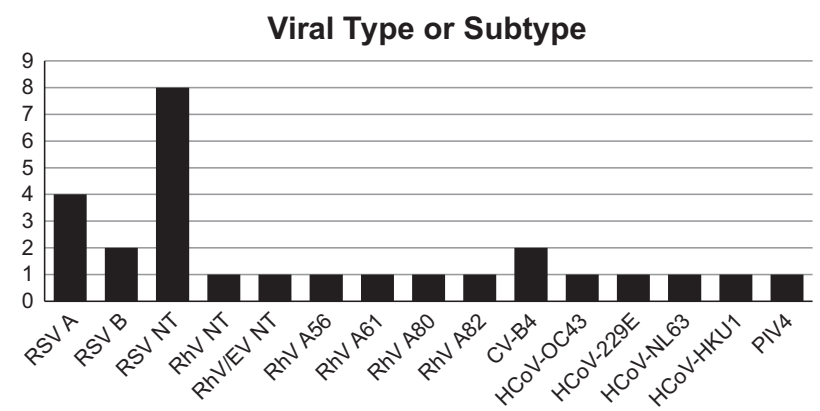

Figure 3. Viral type or subtype. CV, coxsackievirus; EV, enterovirus; HCoV, human coronavirus; PIV parainfluenzavirus; NT, non-typable; RhV, rhinovirus; RSV, respiratory syncytial virus.

Table 3. Presence of bacterial culture $(+)$ infections

\begin{tabular}{lcc}
\hline & $R V P+(\mathrm{N}=29)$ & $R V P-(\mathrm{N}=59)$ \\
\hline Bacterial cultures (+) & $9 / 29(31 \%)$ & $17 / 59(29 \%)$ \\
Blood & $1(3 \%)^{\mathrm{a}}$ & $4(7 \%)^{\mathrm{b}}$ \\
Urine & $1(3 \%)^{\mathrm{c}}$ & $3(5 \%)^{\mathrm{d}}$ \\
Cerebrospinal fluid & $1(3 \%)^{\mathrm{e}}$ & $1(2 \%)^{\mathrm{e}}$ \\
Respiratory & $4(14 \%)^{\mathrm{g}}$ & $9(15 \%)^{\mathrm{h}}$ \\
Wound & $2(7 \%)^{\mathrm{i}}$ & \\
\hline
\end{tabular}

Abbreviations: RVP+, respiratory viral panel positive; RVP -, respiratory viral panel negative. ${ }^{a} \mathrm{MSSA}$, Methicillin-susceptible Staphylococcus aureus baenibacillus; GAS, Group A Streptococcus; Staphylococcus haemolyticus; GBS, Group B Streptococcus. 'GBS. ${ }^{\mathrm{d} E n t e r o b a c t e r ~ c l o a c a e ; ~ E s c h e r i c h i a ~ c o l i ; ~}$

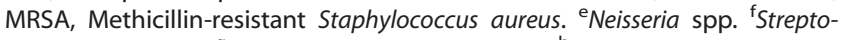
coccus pneumonia. ${ }^{9} \mathrm{MSSA}$; Haemophilus influenza. ${ }^{\mathrm{h}}$ Klebsiella oxytoca; MSSA; Enterobacter cloacae; Pantoea spp.; Acinetobacter; Klebsiella oxytoca; MRSA; Enterobacter spp., MSSA, Klebsiella spp.; Pantoea spp.; MSSA, Klebsiella oxytoca, Stenotrophomonas maltophilia; GBS. 'MSSA.

statistically significant clinical presentations of infants that prompted concern for RVI and with a respiratory virus detected (Table 2).

There were a total of 357 LOS evaluations, 29 (8\%) had a respiratory virus detected. In the 88 evaluations studied with clinical suspicion for RVI, 29 (33\%) were positive for a respiratory virus. There was no difference in the occurrence of RVI between preterm ( 18 of 62 ) and term (11 of 26 ) infants ( 29 vs $42 \%, P=0.23$ ). RSV ( 14 of $29 ; 48 \%$ ) was the predominant virus detected. Figures 2 , 3 depict the different viruses identified in infants with LOS evaluations. Three infants had viral co-infections (RSV-A and RhVA56; RSV non-typable and HCoV-229E; RSV and RhV nontypable) and nine other infants (31\%) had viral-bacterial coinfections outlined in Table 3. In infants without a respiratory virus detected, 17 of 59 (29\%) had a bacterial organism isolated.

There was no significant difference in infants requiring increased respiratory support when a respiratory virus was detected compared to those with a negative respiratory viral PCR panel ( 83 vs $69 \%, P=0.18$ ). However, all but one term infant with RSV infection required increased respiratory support, which included low flow nasal cannula (1), high-flow nasal cannula (4), continuous positive airway pressure (3) and mechanical ventilation (5). There was no difference in the use of mechanical ventilation among infants with RSV detected compared to those with other viruses ( 36 vs $33 \%, P=0.89$ ).

Antimicrobial therapy was withheld or discontinued on most infants with a respiratory virus detected (18 of $29 ; 62 \%)$ and in the majority without a concomitant bacterial infection (18 of 20; $90 \%$ ). In the remaining 11 of 29 infants with a respiratory virus detected, antimicrobial therapy was continued in nine infants for confirmed 
bacterial co-infections and in two infants with clinical presentation of necrotizing enterocolitis and meningitis respectively.

\section{DISCUSSION}

Main findings

In all infants undergoing LOS evaluations, we found that $8 \%$ of those for whom there was concern for respiratory viral infection, had a respiratory virus detected. Apnea was the most common symptom prompting workup in all infants being evaluated for LOS. While the most significant clinical presentations for RVI were cough, nasal congestion, lethargy, poor feeding and increased work of breathing. RSV was the most common respiratory virus isolated, see Figures 2 and 3for further characterization of viruses detected. Antibiotic therapy was withheld or discontinued in $62 \%$ of infants undergoing LOS evaluation.

\section{Interpretation of findings}

The frequency of respiratory viral infections varies in infants undergoing sepsis evaluations. The incidence of viral infections in the NICU has been reported to range from $1 \%^{20}$ to as high as $52 \%$ over a 12-month surveillance period for $\mathrm{RVI}^{5}{ }^{5}$ Our findings corroborate with Ronchi et al. $^{4}$ and Kidszun et al., ${ }^{21}$ in which a respiratory virus was detected in 6 and $6.8 \%$, respectively, in infants undergoing evaluation for LOS. Our study differs in that only 88 of $357(25 \%)$ infants evaluated for LOS also had a respiratory viral panel obtained. In this group, 62 of 88 (70\%) were preterm infants and 18 of $62(29 \%)$ tested positive for a respiratory virus. This is in stark contrast to $52 \%$ of prematurely born infants in the cohort followed by Bennett et al..$^{5}$ Our finding is comparable to the report by Diniz et al. ${ }^{21}$ and Kujari et al., ${ }^{22}$ in which respiratory viruses were diagnosed in 29.5 and $20 \%$, respectively.

A high index of suspicion remains the cornerstone in diagnosing respiratory viral infections in infants, ${ }^{4,23,24,30}$ prompting the use of the viral PCR panel. Similar clinical findings (cough, congestion and increased work of breathing) were described in our study on infants who had a respiratory virus detected; however, non-specific symptoms such as lethargy and poor feeding were also noted among infants with RVI. In comparing preterm infants to term infants with RVI, the presence of apnea (67 vs $9 \%, P<0.01$ ) and the need for increased respiratory support (94 vs $64 \%, P=0.03$ ) were significantly higher in the preterm group, whereas fever ( 0 vs $55 \%, P<0.01$ ) was the predominant symptom in-term infants with RVI. A feasibility study by Kidszun et $a l^{\beta}$ showed no specific clinical characteristics were observed in infants evaluated for nosocomial bacterial sepsis with proven RVI.

In general, the etiology of RVI in infants undergoing sepsis evaluation may vary across different institutions $s^{4,5}$ and during different periods of transmission in the newborn period. ${ }^{3,25}$ Some viral pathogens may predominate depending on the clinical settings: for example an influenza A (H1NI/2009) outbreak in-term and preterm infants with infected healthcare workers as the possible source ${ }^{6}$ or PIV3 and hMPV in premature infants undergoing surveillance for nosocomial RVI. ${ }^{5}$

Certain viral types or subtypes may be associated with disease severity of respiratory viral infections. In a retrospective review by Ronchi et al., ${ }^{11}$ disseminated neonatal adenoviral infection was associated with significant mortality and morbidity. In a recent case report, premature neonate being treated for 'culture-negative sepsis' was diagnosed with disseminated adenoviral infection late in the disease course, which was ultimately fatal ${ }^{22}$ increasing the clinician's awareness of utilizing the viral panel when LOS is suspected. An outbreak of parainfluenza 3 had been reported in seven preterm infants with a spectrum of presentation that consisted of apnea, bradycardia, flu-like illness and progressive respiratory distress. ${ }^{23} \mathrm{RhV}$ has been associated with nosocomial outbreaks in preterm infants causing significant respiratory compromise., ${ }^{1,24,26}$ RSV has been implicated in numerous outbreaks leading to severe disease, including acute respiratory distress syndrome or even death. ${ }^{7}$ There was no evidence of clustering or concern for outbreak of RSV in about half of the respiratory virus cases detected in our study.

Unfortunately, the majority of RSV-positive samples in our study were unable to be typed and we are therefore unable to comment on the distribution between the different subgroups (RSV-A, RSV-non-typable, RSV-B). Bennett et al. found no significant difference between the rates of occurrences of cases across the different subgroups. ${ }^{5}$ Furthermore, most infants with RSV infection required respiratory support, but there was no significant difference in the use of mechanical ventilation compared to those with other viruses detected.

Identification of respiratory viral infections may impact the care and management of infants being evaluated for sepsis. Silva et al. ${ }^{27}$ reported on the impact of the early identification of an index case of RSV, leading to cohorting exposed preterm infants and the use of palivizumab prophylaxis, thus preventing the development of new cases. In an era when there is significant variation in the prescribing practice in the $\mathrm{NICU}^{28}$ microbiologic information may help clinicians in withholding or discontinuing antibiotics. Antibiotic use was withheld or discontinued in the majority $(62 \%)$ of infants undergoing sepsis evaluations and in all infants with limited evaluations when a respiratory virus is detected in our cohort. The use of molecular assays has been recommended to confirm respiratory viral infections in infants $s^{3,4}$ and to assist with the antibiotic stewardship infrastructure in the NICU. ${ }^{12}$ If available in a medical center, respiratory viral PCR test should be used judiciously if the caregiver has clinical suspicion for RVI. ${ }^{4}$

\section{Strengths and limitations}

The major strength of this prospective observational study is utilizing the respiratory viral panel as part of the evaluation for LOS in the NICU. It allowed providers to use their clinical suspicion in using available respiratory viral PCR panel on infants undergoing sepsis evaluations. This study not only confirms the importance of considering RVI among infants undergoing sepsis evaluations ${ }^{4,22,29}$ but demonstrates that in the majority of patients antibiotic therapy was withheld or discontinued when a respiratory virus was detected.

There are several limitations identified in this study. The number of enrolled term infants was below the calculated sample size large enough to detect at least 9\% difference in the incidence of RVI compared to preterm infants (alpha 0.05, beta 0.05). ${ }^{19}$ In contrast to other studies, we included all infants being evaluated for LOS requiring intensive care support rather than limiting to those during their birth hospitalization only. This may explain why we did not find a significant difference in the need for mechanical ventilation in infants when a respiratory virus was detected or not. Increasing the number of infants enrolled involving several medical centers with a NICU would allow examining disease severity associated with specific viral types or subtypes. However, in order to enable such a study, the respiratory viral PCR panel would have to be available in the other medical centers. Another limitation to our study is that we do not know the background prevalence of RVI in our NICU and future surveillance studies may be of benefit.

\section{CONCLUSION}

We conclude that respiratory viral infection should be considered in infants undergoing late-onset sepsis evaluation to limit antibiotic therapy. Especially, among those infants where caregivers have clinical suspicion of viral infections presenting with cough, nasal congestion, lethargy, poor feeding and increased 
work of breathing. Early recognition can prevent viral outbreaks in the NICU, prompt initiation of appropriate antiviral treatment, enable providers to withhold or discontinue inappropriate antibiotics and contribute to NICU-specific stewardship initiatives. Future research should address issues on colonization versus disease-associated RVI in the NICU. Furthermore, multicenter studies with larger sample sizes should investigate the possibility of associations between specific viral types or subtypes and increased disease severity of RVI or various specific syndromes.

\section{CONFLICT OF INTEREST}

The authors declare no conflict of interest.

\section{ACKNOWLEDGEMENTS}

We specially thank Shafiq Butt (AMC Microbiology) for arranging the transfer of respiratory specimens to Wadsworth Center for sequence analyses.

\section{ETHICAL STATEMENT}

The local IRB approved on 07 March 2014 (3769) the single-center prospective observational study through expedited review category 5 with waiver from the Requirement to Obtain Informed Consent (CFR 45.46.116(d)) and Waiver of HIPAA Authorization approved 06 March 2014).

\section{REFERENCES}

1 van Piggelen RO, van Loon AM, Krediet TG, Verboon-Maciolek MA. Human rhinovirus causes severe infection in preterm infants. Pediatr Infect Dis J 2010; 29(4): 364-365.

2 Patel N, The TG. New-onset neonatal pulmonary hypertension associated with a rhinovirus infection. Can Respir J 2012; 19(1): 33-34.

3 Santos RP, Tristram D. A practical guide to the diagnosis, treatment, and prevention of neonatal infections. Pediatr Clin North Am 2015; 62(2): 491-508.

4 Ronchi A, Michelow IC, Chapin KC, Bliss JM, Pugni L, Mosca F et al. Viral respiratory tract infections in the neonatal intensive care unit: the VIRIoN-I study. J Pediatr 2014; 165(4): 690-696.

5 Bennett NJ, Tabarani CM, Bartholoma NM, Wang D, Huang D, Riddell SW et al. Unrecognized viral respiratory tract infections in premature infants during their birth hospitalization: a prospective surveillance study in two neonatal intensive care units. J Pediatr 2012; 161(5): 814-818.

6 Tsagris V, Nika A, Kyriakou D, Kapetanakis I, Harahousou E, Stripeli F et al. Influenza A/H1N1/2009 outbreak in a neonatal intensive care unit. J Hosp Infect 2012; 81(1): 36-40.

7 Kilani RA. Respiratory syncytial virus (RSV) outbreak in the NICU: description of eight cases. J Trop Pediatr 2002; 48(2): 118-122.

8 Kidszun A, Hansmann A, Winter J, Grondahl B, Knuf M, Weise K et al. Detection of respiratory viral infections in neonates treated for suspicion of nosocomial bacterial sepsis: a feasibility study. Pediatr Infect Dis J 2014; 33(1): 102-104.

9 Rogers BB, Shankar P, Jerris RC, Kotzbauer D, Anderson EJ, Watson JR et al. Impact of a rapid respiratory panel test on patient outcomes. Arch Pathol Lab Med 2015; 139(5): 636-641.
10 Popowitch EB, O'Neill SS, Miller MB. Comparison of the biofire filmarray RP, Genmark eSensor RVP, luminex xTAG RVPv1, and luminex xTAG RVP fast multiplex assays for detection of respiratory viruses. J Clin Microbiol 2013; 51(5): 1528-1533.

11 Ronchi A, Doern C, Brock E, Pugni L, Sanchez PJ. Neonatal adenoviral infection: a seventeen year experience and review of the literature. J Pediatr 2014; 164(3): 529-535, e521-524.

12 Cantey JB, Patel SJ. Antimicrobial stewardship in the NICU. Infect Dis Clin North Am 2014; 28(2): 247-261

13 Xu M, Qin X, Astion ML, Rutledge JC, Simpson J, Jerome KR et al. Implementation of filmarray respiratory viral panel in a core laboratory improves testing turnaround time and patient care. Am J Clin Pathol 2013; 139(1): 118-123.

14 Loeffelholz MJ, Pong DL, Pyles RB, Xiong Y, Miller AL, Bufton KK et al. Comparison of the filmarray respiratory panel and prodesse real-time PCR assays for detection of respiratory pathogens. J Clin Microbiol 2011; 49(12): 4083-4088.

15 Babady NE, Mead P, Stiles J, Brennan C, Li H, Shuptar S et al. Comparison of the luminex XTAG RVP fast assay and the Idaho technology filmarray RP assay for detection of respiratory viruses in pediatric patients at a cancer hospital. J Clin Microbiol 2012; 50(7): 2282-2288.

16 Butt SA, Maceira VP, McCallen ME, Stellrecht KA. Comparison of three commercial RT-PCR systems for the detection of respiratory viruses. J Clin Virol 2014; 61(3): 406-410.

17 Nix WA, Oberste MS, Pallansch MA. Sensitive, seminested PCR amplification of VP1 sequences for direct identification of all enterovirus serotypes from original clinical specimens. J Clin Microbiol 2006; 44(8): 2698-2704.

18 Sullender WM, Sun L, Anderson L. Analysis of respiratory syncytial virus genetic variability with amplified cDNAs. J Clin Microbiol 1993; 31(5): 1224-1231.

19 DG U. Uitenbroek DG. Sample Size, Simple Interactive Statistical Analysis - Binomial; 1997. Available at: http://www.quantitativeskills.com/sisa/distributions/ binomial.htm (Accessed 08 November 2013).

20 Verboon-Maciolek MA, Krediet TG, Gerards LJ, Fleer A, van Loon TM. Clinical and epidemiologic characteristics of viral infections in a neonatal intensive care unit during a 12-year period. Pediatr Infect Dis J 2005; 24(10): 901-904.

21 Diniz EM, Vieira RA, Ceccon ME, Ishida MA, Vaz FA. Incidence of respiratory viruses in preterm infants submitted to mechanical ventilation. Rev Inst Med Trop Sao Paulo 2005; 47(1): 37-44.

22 Moallem $M$, Song $E$, Jaggi $P$, Conces MR, Kajon AE, Sanchez PJ. Adenovirus and "Culture-Negative Sepsis" in a preterm neonate. AJP Rep 2016; 6(4): e417-e420.

23 Teo WY, Rajadurai VS, Sriram B. Morbidity of parainfluenza 3 outbreak in preterm infants in a neonatal unit. Ann Acad Med Singapore 2010; 39(11): 837-836.

24 Steiner M, Strassl R, Straub J, Bohm J, Popow-Kraupp T, Berger A. Nosocomial rhinovirus infection in preterm infants. Pediatr Infect Dis J 2012; 31(12): 1302-1304.

25 Santos RPM, MSCS; Tristram, Debra MD. A practical guide to the diagnosis, treatment, and prevention of neonatal infections. Pediatr Clin N Am 2015; 62: 491-508

26 Reid AB, Anderson TL, Cooley L, Williamson J, McGregor AR. An outbreak of human rhinovirus species $C$ infections in a neonatal intensive care unit. Pediatr Infect Dis J 2011; 30(12): 1096-1095.

27 Silva Cde A, Dias L, Baltieri SR, Rodrigues TT, Takagi NB, Richtmann R. Respiratory syncytial virus outbreak in neonatal intensive care unit: Impact of infection control measures plus palivizumab use. Antimicrob Resist Infect Control 2012; 1(1): 16

28 Schulman J, Dimand RJ, Lee HC, Duenas GV, Bennett MV, Gould JB. Neonatal intensive care unit antibiotic use. Pediatrics 2015; 135(5): 826-833.

29 Kidszun A, Klein L, Winter J, Schmeh I, Grondahl B, Gehring S et al. Viral infections in neonates with suspected late-onset bacterial sepsis-A Prospective Cohort Study. Am J Perinatol 2016; 34 (1):1-7. 